\title{
Feasibility of Use of a Barbed Suture (V-Loc 180) for Quilting the Donor Site in Latissimus Dorsi Myocutaneous Flap Breast Reconstruction
}

\author{
Dinesh Kumar Thekkinkattil, Tasadooq Hussain, Tapan Kumar Mahapatra, \\ Penelope Louise McManus, Peter John Kneeshaw \\ Department of Oncoplastic Breast Surgery, Castle Hill Hospital, Hull and East Yorkshire Hospitals NHS Trust, Cottingham, East Yorkshire, UK
}

Background Latissimus dorsi (LD) myocutaneous flap is a popular method of breast reconstruction which can be associated with high incidence of seroma formation. Quilting sutures at the harvest site are used to reduce this. Barbed sutures are self anchoring sutures which avoid multiple knotting and can be useful in quilting.

Methods A retrospective analysis of prospectively maintained database of patients who underwent LD flap breast reconstruction between January 2009 and January 2011 was carried out. Seroma formation at the harvest site, wound related complications, inpatient stay and duration of surgery were analysed and a comparison was made between two groups where quilting was done with barbed (V-Loc) suture and conventional polydioxanone (PDS) II sutures. Results Fifty-seven patients were included of which 33 had quilting by V-Loc sutures and in 24 patients PDS II suture was used. Median age in the PDS group was 55 years (interquartile range [IQR)], 45 to 61 years) which was comparable to the V-Loc group (53 years [IQR, 48 to 59 years]; P-value 0.948$)$. Sixteen patients $(28 \%)$ had significant seroma formation and $5(9 \%)$ patients developed superficial wound dehiscence. Incidences of seroma or wound complications were comparable ( $\mathrm{P}$-value 0.378 and 1.00 , respectively). Secondary outcomes such as total duration of surgery, total inpatient stay, total amount of drain at the donor site were also similar in two groups.

Conclusions Use of barbed sutures for quilting the donor site in LD flap reconstruction is a feasible option and the associated seroma formation and wound complications are comparable with conventional sutures.

Keywords Barbed suture / V-Loc / Quilting / Seroma / Latissimus dorsi flap
Correspondence:

Dinesh Kumar Thekkinkattil

Department of Oncoplastic Breast

Surgery, Castle Hill Hospital, Castle

Road, Cottingham, East Yorkshire,

HU16 5JQ, UK

Tel: $+44-1482624238$

Fax: +44-1482622650

E-mail:drdiku@gmail.com

This article was presented at the Oncoplastic and Reconstructive Breast Surgery on September 26-28, 2011 in Nottingham, United Kingdom.

No potential conflict of interest relevant to this article was reported.

\section{INTRODUCTION}

The latissimus dorsi myocutaneous flap is a popular method of breast reconstruction as it is a reliable flap with a consistent vascular pedicle and adequate volume can be harvested to perform either an autologous or implant assisted reconstruction. One of the major drawbacks with this flap is the incidence of donor site complications mainly seroma formation. The incidence of seroma at the harvest site can be as high as $70 \%$ to $80 \%$ [1-3]. Various techniques have been used to reduce the seroma forma-

Copyright ( $\odot 2013$ The Korean Society of Plastic and Reconstructive Surgeons

This is an Open Access article distributed under the terms of the Creative Commons Attribution Non-Commercial License (http://creativecommons.org/

licenses/by-nc/3.0/) which permits unrestricted non-commercial use, distribution, and reproduction in any medium, provided the original work is properly cited.

www.e-aps.org 
tion such as quilting sutures, fibrin glue, pressure dressing, triamcinolone injections [4-7]. These techniques are used either in isolation or in various combinations. Most of the time a seroma is asymptomatic but it may predispose to infection and subsequent wound complications $[2,3,8]$.

There are various risk factors for seroma formation and most of the time it is multifactorial in origin such as disrupted lymphatic and vascular channels, dead space, inflammatory mediators and creation of large raw surfaces $[9,10]$. Quilting sutures are intended to reduce the dead space and in turn a reduction in seroma formation [11]. Fibrin glue sealants help to obliterate the dead space reduces the serous exudates by sealing the lymphatics [12]. On the contrary, some of the studies suggest that fibrin glue is not useful in this regard $[6,13]$.

Prevention of seroma formation and related wound complications can avoid delay in delivering adjuvant therapy to breast cancer patients especially in the immediate reconstruction settings [14]. Many studies have proven that quilting of the skin flaps at the harvest site in latissimus dorsi flap breast reconstruction reduces the seroma formation and subsequent complications [11].

Quilting suturing is usually carried out using absorbable sutures. An ideal suture should be easy to handle, easy to perform, providing optimal apposition with sufficient strength. Placing interrupted sutures and tying multiple knots in limited space between the skin flap and the chest wall fascia can be challenging and time consuming for surgeons while quilting the harvest site of latissimus dorsi flap in breast reconstruction.

Barbed sutures are gaining popularity in many surgical fields in the recent times even though the concept dates back to 1951 when the idea of using barbed sutures for tendon repair was presented $[15,16]$. Barbed sutures are available in absorbable or non absorbable monofilament sutures which have got axially placed barbed segments along its length. These barbs can be unidirectional or bi-directional. These sutures are in use in many surgical fields such as plastic surgery, urology and gynaecology for many years. They are self anchoring and avoid the need for multiple knot tying and are thought to be 50\% quicker than suturing with conventional suture materials. Weakest point in a suture line is the knot and with studies showing a reduction in tensile strength from $35 \%$ to $95 \%[16,17]$. The volume of the knot is directly proportional to the surrounding tissue inflammation [18] Barbed sutures are also helpful in distributing the tension equally along the wound and also avoid the need for extra hands while suturing $[19,20]$.

\section{Aim}

The primary aim of the study was to assess the feasibility of use of barbed V-Loc 180 (Covedian, Mansfied, MA, USA) sutures in quilting the back wound and compare the incidence of wound complications with that of conventional quilting sutures with $2 \mathrm{O}$ polydioxanone (PDS) II (Ethicon Inc., Somerville, NJ, USA).

Secondary aims were to compare the total duration of surgery, total inpatient stay and cost incurred among the two groups.

\section{METHODS}

This was a retrospective study of a prospectively maintained data over duration of 2 years between January 2009 and January 2011. The duration was selected as the suture used to quilt the back wound in latissimus dorsi flap reconstruction was changed from absorbable 2 O polydioxanone PDS II (Ethicon) to V-Loc 180 (Covedian) absorbable barbed sutures in 2010. Demographic data was collected from the notes. We also collected perioperative data such as, type of surgery, associated procedures, total duration of operation, type of suture used to quilt the back wound, total inpatient stay, total drainage from the breast drain and back drains before they have been removed.

Postoperative complication details were collected from clinic letters and dressing clinic attendance documentation. Data were collected regarding the incidence of significant seroma, wound status and management of wound complications if any. A significant seroma was defined as those which needed aspiration because of patient discomfort or pressure effect. In a small subset of patients we calculated the time spent on quilting stitches.

\section{Patients}

Patients who underwent an autologous or implant assisted latissimus dorsi flap breast reconstruction between a period of January 2009 and January 2011 were included. Both immediate and

Fig. 1. V-Loc suture with welded loop and barbs

V-Loc suture device with axial barbs and welded loop at the end.

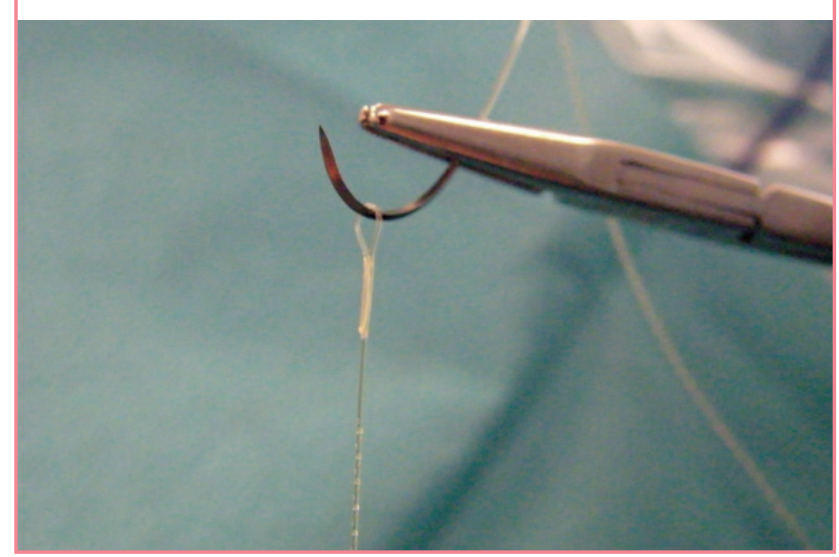


delayed reconstructions were included. All the flaps were raised by dissection with electrocautery and bipolar dissecting scissors near the pedicle. The skin incision was horizontal or oblique to include a fusiform skin paddle. We infiltrated the subcutaneous plane with saline and adrenaline solution before raising the flap.

In the earlier half of the study, we were using interrupted $2 \mathrm{O}$ PDS II (Ethicon) sutures for quilting. Multiple rows of sutures were used. This suture was replaced by $3 \mathrm{O}$ V-Loc (Covedian) sutures for quilting from 2010 onwards (Fig. 1). The method of quilting was by horizontal layers of continuous sutures with V-Loc. The welded loop design and the barbs of V-Loc suture eliminate the need for tying any knots while quilting (Fig. 2). In both groups the skin was closed in two layers: a deep layer of 2 O PDS II and a superficial subcuticular layer of $3 \mathrm{O}$ Monocryl (poliglecaprone, Ethicon). Two suction drains were used in all cases (either a No 10 Blake [Ethicon] drain or a Redi-vac drain), one in the back wound at donor site and second one in the breast. Drains were removed when the drainage over 24 hours were $<50 \mathrm{~mL}$ or total number of days were 7 days whichever is earlier.

\section{Statistical analysis}

The statistical analysis was carried out by using PASW ver. 18.0.0 (IBM SPSS, Armonk, NY, USA). P-value of $<0.05$ was deemed significant. Categorical variables were analysed by chi-square test and Fisher's exact test was used when the frequency was small.

\section{Fig. 2. Quilting by horizontal layers of V-Loc suture}

Quilting procedure with V-Loc suture device by placing the continuous sutures in horizontal layers.

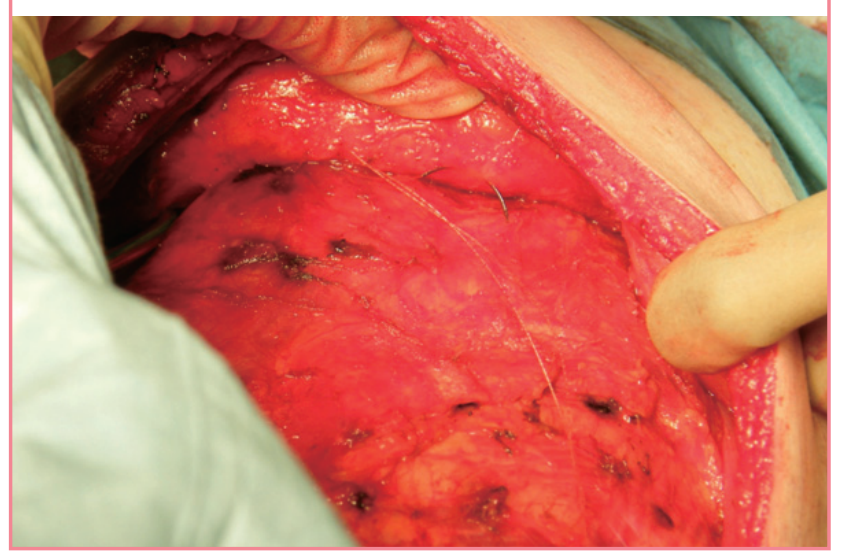

Mann Whitney $\mathrm{U}$ test was used to analyse quantitative variables between two groups. A logistic regression analysis was carried out to identify factors that were associated with significant seroma formation and donor site wound dehiscence (age, duration of surgery, mastectomy specimen weight, total volume of drainage in the back drain, radiotherapy and type of reconstruction).

\section{RESULTS}

We had a total of 70 patients who underwent latissimus dorsi flap reconstruction during the study period. Only 57 patients had complete data available and hence were included in the study. Of these 33 patients had quilting by V-Loc sutures and rest 24 patients had the quilting done by PDS II suture. The median age in the PDS group was 55 (interquartile range [IQR], 45 to $61)$ years. This was comparable to the median age of patients in the V-Loc group ( 53 years; IQR, 48 to 59 years) (P-value 0.948, Kruskal Wallis test).

Thirty patients (53\%) underwent immediate reconstruction and 27 patients (47\%) had the reconstruction as a delayed procedure. Fourteen patients in the PDS group and 13 patients in VLoc group underwent delayed reconstruction while 10 patients in the PDS group and 20 patients in the V-Loc group had immediate breast reconstruction. Even though the number of skin sparing mastectomies performed along with flap reconstruction were double ( 20 vs. 10 ) in the V-Loc group, the difference failed to achieve any statistical significance (Table 1) (P-value 0.157, chi-square test). Three patients in the delayed reconstruction group had their expander or previous implant removed. One patient had implant assisted latissimus dorsi flap reconstruction as immediate procedure. 3 patients had axillary node clearance along with flap reconstruction. Twenty two (39\%) patients had previous radiotherapy to the chest wall.

Table 1. Comparison of type of reconstruction

\begin{tabular}{|c|c|c|c|}
\hline \multirow{2}{*}{ Type of reconstruction } & \multicolumn{2}{|c|}{ Suture } & \multirow{2}{*}{ P-value ${ }^{a)}$} \\
\hline & PDS & V-Loc & \\
\hline Immediate $(n=30)$ & 10 & 20 & 0.157 \\
\hline Delayed $(n=27)$ & 14 & 13 & \\
\hline
\end{tabular}

Table 2. Incidence of seroma and superficial wound dehiscence in the two groups

\begin{tabular}{|c|c|c|c|c|c|c|}
\hline \multirow{2}{*}{ Type of suture } & \multicolumn{2}{|c|}{ Seroma formation } & \multirow{2}{*}{ P-value ${ }^{\text {a) }}$} & \multicolumn{2}{|c|}{ Superficial wound dehiscence } & \multirow{2}{*}{ P-value ${ }^{a)}$} \\
\hline & Yes & No & & Yes & No & \\
\hline PDS $(n=24)$ & 5 & 19 & 0.378 & 2 & 22 & 1.00 \\
\hline V-Loc $(n=33)$ & 11 & 22 & & 3 & 30 & \\
\hline
\end{tabular}


Table 3. Comparison of age, duration of surgery, in patients stays and drain volumes in two suture groups

\begin{tabular}{|lccccc|}
\hline Type of suture & Age $(\mathrm{yr})$ & $\begin{array}{c}\text { Length of inpatient } \\
\text { stay (day) }\end{array}$ & $\begin{array}{c}\text { Total duration of } \\
(\mathbf{m i n})\end{array}$ & $\begin{array}{c}\text { Total volume in the } \\
\text { breast drain }(\mathbf{m L})\end{array}$ & $\begin{array}{c}\text { Total volume in the } \\
\text { back drain }(\mathbf{m L})\end{array}$ \\
\hline PDS II & $55(45-61)$ & $5(5-7)$ & $319(282-345)$ & $50(10-115)$ & $695(385-1,195)$ \\
V-LoC & $53(48-59)$ & $6(5-7)$ & $311(300-335)$ & $60(20-170)$ & $700(430-879)$ \\
P-value ${ }^{\text {a) }}$ & 0.948 & 0.329 & 0.881 & 0.382 & 0.790 \\
\hline $\begin{array}{l}\text { Values are presented as median (interquartile range) } \\
\text { alMann-Whitney U test. }\end{array}$ & & & & \\
\hline
\end{tabular}

Table 4. Correlation of total volume in the back drain and subsequent seroma formation

\begin{tabular}{|c|c|c|c|}
\hline \multirow{2}{*}{$\begin{array}{l}\text { Total volume in back } \\
\text { drain }(\mathrm{mL})\end{array}$} & \multicolumn{2}{|c|}{ Seroma formation } & \multirow{2}{*}{ P-value ${ }^{a)}$} \\
\hline & Yes & No & \\
\hline$\leq 1,000$ & 11 & 32 & 0.505 \\
\hline$>1,000$ & 5 & 9 & \\
\hline
\end{tabular}

Sixteen (28\%) patients in our study series developed significant seroma which needed aspiration and 5 (9\%) patients developed superficial wound dehiscence (Table 2). The incidences of seroma formation or wound complications were not significantly different in the two groups (P-value 0.378 and 1.00, respectively; Fisher's exact test).

Secondary outcomes measured included the total duration of surgery, total inpatient stay, total volume in the breast drain and drain at the harvest site. Table 3 shows that there was no significant difference in these variables in the two suture groups.

A logistic regression analysis did not show any significant association between age, weight of mastectomy specimen, type of suture, duration or type of surgery, length of inpatient stay, total drain volume in the back drain or history of previous radiotherapy and subsequent seroma formation or wound dehiscence.

There was no significant correlation between total amount of drainage in the back drain and subsequent seroma formation (Table 4) (P-value 0.505, Fisher's exact test).

The mastectomy weight details were available in 40 of our patients and there was no significant difference between the two groups of patients who developed significant seroma and those without (median weight of $649 \mathrm{~g}$ [IQR, 350 to $970 \mathrm{~g}]$ vs. $429 \mathrm{~g}$ [IQR, 244 to $855 \mathrm{~g}$ ]; Mann Whitney U test 0.282).

Average number of V-Loc sutures used to quilt the back wound was 4 and in a small subset of patients who underwent quilting by V-Loc sutures $(n=10)$ the median time to perform quilting suturing was 20 minutes.

The cost of a single pack of $3 \mathrm{O}$ V-Loc (Covedian) suture to the trust was $£ 20$ while a single pack of 2 O PDS II (Ethicon) suture cost the trust $£ 4$.

\section{DISCUSSION}

The incidence of seroma rate (28\%) in our study group was much lower than in other comparable groups but was similar to reported incidence in the randomized trial by Menke et al. $[3,8,21]$. This reiterates the findings in various studies showing reduced incidence of seroma formation with quilting sutures [11]. Various suture materials and the techniques of suturing has been extensively studied in the past $[19,22]$. Barbed sutures are another addition to surgeon's armamentarium and are gaining popularity in various surgical fields $[16,23]$. Knotless suturing is very useful in laparoscopic and robotic surgical procedures and avoids inconvenience of placing multiple knots as well as eliminates loss suture strength at the site of knots [24]. Self anchoring barbed sutures also eliminate the need for additional assistance in following sutures and tend to distribute tension along the length of the wound $[16,25]$.

In our series, we did not calculate the amount of time required to perform the quilting. But the total duration of surgery was similar in the two suture groups. This could well be due to the fact that there was almost double the number of skin sparing mastectomies (20 vs. 10) performed in the V-Loc group when compared with the PDS II group. Even though this observation failed to achieve any statistical significance, this could have negated the advantage gained by quicker quilting by barbed sutures.

The incidence of superficial wound dehiscence is similar in both suture groups. None of these patients needed any surgical intervention and wounds were managed by regular dressings. Similarly the total duration of inpatient stay and drainage from the breast and harvest site drains were similar in the two groups. We divided the patients into two groups depending upon the total amount of drainage from the back drain $(\leq 1,000 \mathrm{~mL}$ vs. $>1,000 \mathrm{~mL}$ total drainage). The seroma formation was not significantly different in these two groups (P-value 0.505 , Fisher's exact test). This justifies our practise of removing the back drain at 7 th postoperative day irrespective of the drainage.

The average number of V-Loc sutures, used per patient was 4 and this cost $£ 80$ to the trust. The number of PDS II sutures used per patient was variable as more sutures were used by sur- 
geons who were performing hand tying. The cost per pack of 2 O PDS II was $£ 4$ to the trust. But all surgeons found the barbed suture easy and convenient to handle while quilting. The need for greater number of PDS II sutures reduces the expenditure difference between the two suture groups.

In our study, quilting by conventional PDS II sutures was done by interrupted sutures. But a continuous suturing technique was used when we used V-Loc barbed sutures. It is unlikely that this difference in technique would have influenced the observed outcome. Other limitations of the study include the retrospective nature of the study and ideally we would like to perform a prospective randomised control trial. Secondly, time taken to perform the quilting part of surgery was not calculated separately from the total duration of the surgery. The extra time taken to carry out the other parts of surgery such as skin sparing mastectomy could have negated the advantage gained by lesser time for quilting with V-Loc sutures as there was a disparity in number of patients underwent skin sparing mastectomy in two groups. Similarly, the lower number of skin sparing mastectomies in the PDS suture group (higher proportion of delayed reconstruction) patients is unlikely to influence the rate of incidence of donor site wound complications as our practice is to design the skin paddle in such a way that excess tension is avoided across the wound and we used implants or planned future sessions of fat transfer to improve the volume of reconstructed breast.

Barbed sutures are feasible option for use in quilting the harvest site in latissimus dorsi flap breast reconstruction. The associated wound related complications such as seroma formation and wound dehiscence are similar and comparable with conventional sutures. A prospective randomised controlled study is needed to validate these observations.

\section{REFERENCES}

1. Tomita K, Yano K, Masuoka T, et al. Postoperative seroma formation in breast reconstruction with latissimus dorsi flaps: a retrospective study of 174 consecutive cases. Ann Plast Surg 2007;59:149-51.

2. Bailey SH, Oni G, Guevara R, et al. Latissimus dorsi donorsite morbidity: the combination of quilting and fibrin sealant reduce length of drain placement and seroma rate. Ann Plast Surg 2012;68:555-8.

3. Clough KB, Louis-Sylvestre C, Fitoussi A, et al. Donor site sequelae after autologous breast reconstruction with an extended latissimus dorsi flap. Plast Reconstr Surg 2002;109: 1904-11.

4. Rios JL, Pollock T, Adams WP Jr. Progressive tension sutures to prevent seroma formation after latissimus dorsi harvest.
Plast Reconstr Surg 2003;112:1779-83.

5. Weinrach JC, Cronin ED, Smith BK, et al. Preventing sero$\mathrm{ma}$ in the latissimus dorsi flap donor site with fibrin sealant. Ann Plast Surg 2004;53:12-6.

6. Cha HG, Kang SG, Shin HS, et al. Does fibrin sealant reduce seroma after immediate breast reconstruction utilizing a latissimus dorsi myocutaneous flap? Arch Plast Surg 2012;39:504-8.

7. Shin IS, Lee DW, Lew DH. Efficacy of quilting sutures and fibrin sealant together for prevention of seroma in extended latissimus dorsi flap donor sites. Arch Plast Surg 2012;39: 509-13.

8. Menke H, Erkens M, Olbrisch RR. Evolving concepts in breast reconstruction with latissimus dorsi flaps: results and follow-up of 121 consecutive patients. Ann Plast Surg 2001; 47:107-14.

9. Bacilious N, Kulber DA, Peters ED, et al. Harvesting of the latissimus dorsi muscle: a small animal model for seroma formation. Microsurgery 1995; 16:646-9.

10. Jain PK, Sowdi R, Anderson AD, et al. Randomized clinical trial investigating the use of drains and fibrin sealant following surgery for breast cancer. Br J Surg 2004;91:54-60.

11. Daltrey I, Thomson H, Hussien M, et al. Randomized clinical trial of the effect of quilting latissimus dorsi flap donor site on seroma formation. Br J Surg 2006;93:825-30.

12. Sierra DH, Feldman DS, Saltz R, et al. A method to determine shear adhesive strength of fibrin sealants. J Appl Biomater 1992;3:147-51.

13. Cipolla C, Fricano S, Vieni S, et al. Does the use of fibrin glue prevent seroma formation after axillary lymphadenectomy for breast cancer? A prospective randomized trial in 159 patients. J Surg Oncol 2010;101:600-3.

14. Callaghan CJ, Couto E, Kerin MJ, et al. Breast reconstruction in the United Kingdom and Ireland. Br J Surg 2002;89: 335-40.

15. McKenzie AR. An experimental multiple barbed suture for the long flexor tendons of the palm and fingers: preliminary report. J Bone Joint Surg Br 1967;49:440-7.

16. Greenberg JA. The use of barbed sutures in obstetrics and gynecology. Rev Obstet Gynecol 2010;3:82-91.

17. Kim JC, Lee YK, Lim BS, et al. Comparison of tensile and knot security properties of surgical sutures. J Mater Sci Mater Med 2007;18:2363-9.

18. Molokova OA, Kecherukov AI, Aliev F, et al. Tissue reactions to modern suturing material in colorectal surgery. Bull Exp Biol Med 2007; 143:767-70.

19. Murtha AP, Kaplan AL, Paglia MJ, et al. Evaluation of a novel technique for wound closure using a barbed suture. Plast Re- 
constr Surg 2006;117:1769-80.

20. Parikh PM, Davison SP, Higgins JP. Barbed suture tenorrhaphy: an ex vivo biomechanical analysis. Plast Reconstr Surg 2009; 124:1551-8.

21. Delay E, Gounot N, Bouillot A, et al. Autologous latissimus breast reconstruction: a 3-year clinical experience with 100 patients. Plast Reconstr Surg 1998;102:1461-78.

22. Hogstrom H, Haglund U, Zederfeldt B. Tension leads to increased neutrophil accumulation and decreased laparotomy wound strength. Surgery 1990;107:215-9.

23. Mulholland RS, Paul MD. Lifting and wound closure with barbed sutures. Clin Plast Surg 2011;38:521-35.

24. van Rijssel EJ, Brand R, Admiraal C, et al. Tissue reaction and surgical knots: the effect of suture size, knot configuration, and knot volume. Obstet Gynecol 1989;74:64-8.

25. Rosen $\mathrm{AD}$. Use of absorbable running barbed suture and progressive tension technique in abdominoplasty: a novel approach. Plast Reconstr Surg 2010;125:1024-7. 